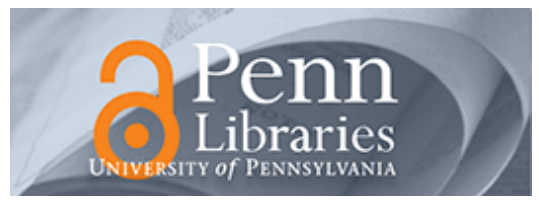

University of Pennsylvania

ScholarlyCommons

Statistics Papers

Wharton Faculty Research

2-1990

\title{
Sets Uniquely Determined by Projections on Axes I. Continuous
}

Case

P. C. Fishburn

J. C. Lagarias

J. A. Reeds

Larry A. Shepp

University of Pennsylvania

Follow this and additional works at: https://repository.upenn.edu/statistics_papers

Part of the Applied Mathematics Commons, and the Statistics and Probability Commons

\section{Recommended Citation}

Fishburn, P. C., Lagarias, J. C., Reeds, J. A., \& Shepp, L. A. (1990). Sets Uniquely Determined by Projections on Axes I. Continuous Case. SIAM Journal on Applied Mathematics, 50 (1), 288-306. http://dx.doi.org/ $10.1137 / 0150017$

This paper is posted at ScholarlyCommons. https://repository.upenn.edu/statistics_papers/413

For more information, please contact repository@pobox.upenn.edu. 


\title{
Sets Uniquely Determined by Projections on Axes I. Continuous Case
}

\begin{abstract}
This paper studies sets $S$ in $\mathrm{R}^{\mathrm{n}}$ which are uniquely reconstructible from their hyperplane integral projections $\mathrm{P}_{\mathrm{i}}\left(\mathrm{x}_{\mathrm{i}} ; \mathrm{S}\right)=\iint \ldots \int X_{S}\left(\left\{\mathrm{x}_{1}, \ldots, \mathrm{x}_{\mathrm{i}}, \ldots, \mathrm{x}_{\mathrm{n}}\right) \mathrm{dx} \mathrm{x}_{1} \ldots \mathrm{dx_{i } - 1} d \mathrm{dx}_{\mathrm{i}}+1 \ldots \mathrm{dx_{n }}\right.$ onto the $n$ coordinate axes of $R^{n}$. It is shown that any additive set $S=\left\{x=\left(x_{1}, \ldots, x_{n}\right): \sum i=1^{n} f_{i}\left(x_{i}\right) \geqq 0\right\}$, where each $f_{i}\left(x_{i}\right)$ is a bounded measurable function, is uniquely reconstructible. In particular, balls are uniquely reconstructible. It is shown that in $R^{2}$ all uniquely reconstructible sets are additive. For $n \geqq 3$, Kemperman has shown that there are uniquely reconstructible sets in $R^{n}$ of bounded measure that are not additive. It is also noted for $n \geqq 3$ that neither of the properties of being additive and being a set of uniqueness is closed under monotone pointwise limits.
\end{abstract}

A necessary condition for $S$ to be a set of uniqueness is that $S$ contain no bad configuration. A bad configuration is two finite sets of points $T_{1}$ in $\operatorname{Int}(S)$ and $T_{2}$ in $\operatorname{Int}\left(S^{c}\right)$, where $S^{C}=R^{n}-S$, such that $T_{1}$ and $T_{2}$ have the same number of points in any hyperplane $x_{i}=c$ for $1 \leqq i \leqq n$, and all $c \in R^{2}$. We show that this necessary condition is sufficient for uniqueness for open sets $S$ in $\mathrm{R}^{2}$.

The results show that prior information about a density $f$ in $\mathrm{R}^{2}$ to be reconstructed in tomography (namely if $f$ is known to have only values 0 and 1 ) can sometimes reduce the problem of reconstructing $f$ to knowing only two projections of $f$. Thus even meager prior information can in principle be of enormous value in tomography.

\section{Keywords}

projections, uniqueness, additive sets, probability

Disciplines

Applied Mathematics | Statistics and Probability 


\title{
SETS UNIQUELY DETERMINED BY PROJECTIONS ON AXES I. CONTINUOUS CASE*
}

\author{
P. C. FISHBURN $\dagger$, J. C. LAgARIAS $\dagger$, J. A. REEDS $\dagger$, AND L. A. SHEPP $\dagger$
}

\begin{abstract}
This paper studies sets $S$ in $\mathbb{R}^{\prime \prime}$ which are uniquely reconstructible from their hyperplane integral projections $P_{i}\left(x_{i} ; S\right)=\iint \cdots \int \chi_{S}\left(x_{1}, \cdots, x_{i}, \cdots, x_{n}\right) d x_{1} \cdots d x_{i-1} d x_{i+1} \cdots d x_{n}$ onto the $n$ coordinate axes of $\mathbb{R}^{n}$. It is shown that any additive set $S=\left\{\mathbf{x}=\left(x_{1}, \cdots, x_{n}\right): \sum_{i=1}^{n} f_{i}\left(x_{i}\right) \geqq 0\right\}$, where each $f_{i}\left(x_{i}\right)$ is a bounded measurable function, is uniquely reconstructible. In particular, balls are uniquely reconstructible. It is shown that in $\mathbb{R}^{2}$ all uniquely reconstructible sets are additive. For $n \geqq 3$, Kemperman has shown that there are uniquely reconstructible sets in $\mathbb{R}^{n}$ of bounded measure that are not additive. It is also noted for $n \geqq 3$ that neither of the properties of being additive and being a set of uniqueness is closed under monotone pointwise limits.

A necessary condition for $S$ to be a set of uniqueness is that $S$ contain no bad configuration. A bad configuration is two finite sets of points $T_{1}$ in Int $(S)$ and $T_{2}$ in Int $\left(S^{c}\right)$, where $S^{c}=\mathbb{R}^{n}-S$, such that $T_{1}$ and $T_{2}$ have the same number of points in any hyperplane $x_{i}=c$ for $1 \leqq i \leqq n$, and all $c \in \mathbb{R}$. We show that this necessary condition is sufficient for uniqueness for open sets $S$ in $\mathbb{R}^{2}$.

The results show that prior information about a density $f$ in $\mathbb{R}^{2}$ to be reconstructed in tomography (namely if $f$ is known to have only values 0 and 1) can sometimes reduce the problem of reconstructing $f$ to knowing only two projections of $f$. Thus even meager prior information can in principle be of enormous value in tomography.
\end{abstract}

Key words. projections, uniqueness, additive sets, probability

AMS(MOS) subject classifications. 05B, 53A, 60B, 62H

1. Introduction. Horn [4] asked in connection with a problem of robot vision whether the unit disk $D$ in $\mathbb{R}^{2}$ is uniquely determined (up to null sets) by its line integral projections onto the $x$ and $y$ axes. At first it may seem unlikely that only two projections of the density

$$
f_{D}(x, y)=\chi_{D}(x, y)= \begin{cases}1 & \text { if }(x, y) \in D \\ 0 & \text { if }(x, y) \notin D\end{cases}
$$

could determine $f(x, y)$ uniquely, but in fact Horn's question has an affirmative answer despite such tempting "counterexamples" as Fig. 1.1. Here is a simple proof: Any two sets having the same projections on the $x$ and $y$ axes have the same area and moment of inertia. The disk $D$ has the minimum moment of inertia among all sets $S$ having the same area as $D$, so that the area and moment of inertia of a disk $D$ characterize it uniquely (up to a null set).

This problem is a special kind of tomographic reconstruction problem in which we attempt to reconstruct a density $f(x, y)$, given two projections and the prior information that the density $f(x, y)$ only takes the values 0 or 1 . For an arbitrary density $f(x, y)$ we generally need to know infinitely many projections to reconstruct $f(x, y)$ uniquely. However, the disk example shows that the prior information that the density $f(x, y)$ is an indicator function can greatly influence the number of projections needed for tomographic reconstruction.

More generally, we consider as given data for reconstructing a set $S$ in $\mathbb{R}^{n}$ the hyperplane integral projections

$$
P_{\mathrm{v}}(t ; S)=\int_{H_{1}(\mathbf{v})} \chi_{S}(\mathbf{x}) d H_{t}, \quad-\infty<t<\infty
$$

* Received by the editors August 10, 1987; accepted for publication (in revised form) January 20, 1989.

$\dagger$ AT\&T Bell Laboratories, Murray Hill, New Jersey 07974. 


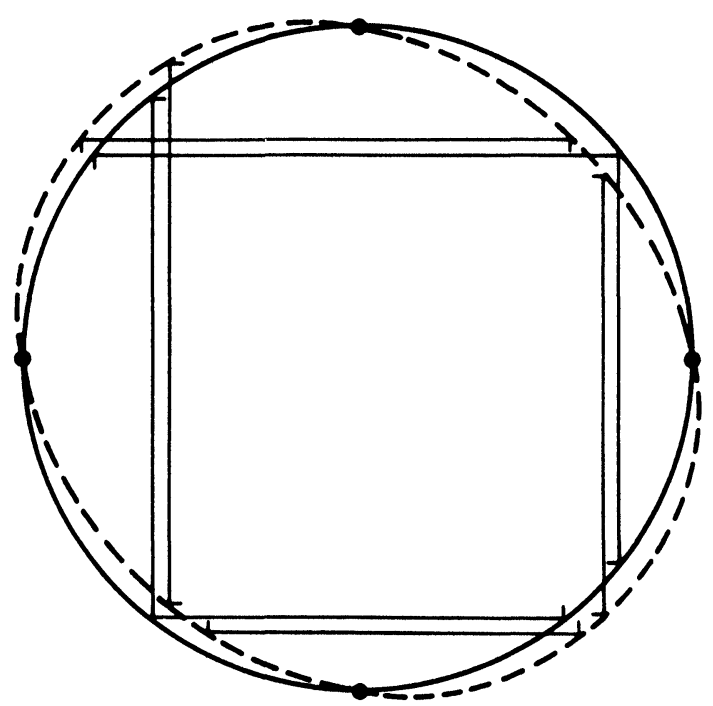

FIG. 1.1. The solid disk is extended out in quadrants II and IV and in in quadrants I and III. The dashed warped disk cannot have the same projections as the original.

where $\chi_{S}(\mathbf{x})$ is the characteristic function of $S, H_{t}(\mathbf{v})$ denotes a hyperplane with normal unit vector $\mathbf{v}$ given by

$$
H_{t}(\mathbf{v})=\{\mathbf{x}:\langle\mathbf{v}, \mathbf{x}\rangle=t\}
$$

and $d H_{t}$ is Lebesgue $(n-1)$-dimensional measure on $H_{t}$. We call the set $\left\{P_{\mathrm{v}}(t ; S):-\infty<\right.$ $t<\infty\}$ the projection onto the line $L_{\mathrm{v}}=\{t \mathrm{v}:-\infty<t<\infty\}$ and associate $P_{\mathrm{v}}(t ; S)$ to the point $t \mathbf{v}$ on this line.

This paper examines the problem of characterizing sets $S$ of finite volume in $\mathbb{R}^{n}$ that are reconstructible (up to null sets) from their projections onto the $n$ coordinate axes of $\mathbb{R}^{n}$, i.e., from

$$
P_{i}\left(x_{i} ; S\right)=\int \cdots \int \chi_{S}\left(x_{1}, \cdots, x_{i}, \cdots, x_{n}\right) d x_{1} \cdots d x_{i-1} d x_{i+1} \cdots d x_{n}
$$

for $-\infty<x_{i}<\infty$ and $1 \leqq i \leqq n$. We call the problem of characterizing sets of uniqueness the continuous unique reconstruction problem. We show that such sets $S$ exist. The number $n$ is the minimal number of directions of projection on lines for which there is a set $S$ (not a null set) such that unique reconstruction of $S$ is possible. To see this, note that if projections in $n-1$ or fewer directions are given, then there is a direction orthogonal to all these directions, and we recover no information about the distribution of the mass of $S$ in this direction, so that unique reconstruction is impossible. Furthermore, this argument shows that for unique reconstruction from projections onto $n$ lines to be possible these lines must lie in $n$ linearly independent directions. In this case we may, without loss of generality, reduce the general problem to the special case (1.1) where the lines are the coordinate axes by an invertible linear transformation of coordinates.

There is an analogous discrete version of the problem which we call the discrete unique reconstruction problem. The discrete problem is to characterize which finite subsets $S$ of the integer lattice points $\mathbb{Z}^{n}$ given by their indicator function

$$
f_{S}\left(x_{1}, \cdots, x_{n}\right)= \begin{cases}1 & \text { if }\left(x_{1}, \cdots, x_{n}\right) \in S, \\ 0 & \text { if }\left(x_{1}, \cdots, x_{n}\right) \notin S,\end{cases}
$$


are uniquely reconstructible from the projections $\left\{P_{i}: 1 \leqq i \leqq n\right\}$, where

$$
P_{i}\left(x_{i}\right)=\sum_{\substack{\left(y_{1}, \cdots, y_{n}\right) \in \mathbb{Z}^{n} \\ y_{i}=x_{i}}} f_{S}\left(y_{1}, \cdots, y_{n}\right) .
$$

The discrete problem is studied in a companion paper [1].

The problem of characterizing sets of uniqueness was studied in the twodimensional case by Lorentz [9] in 1948 in an elegant but apparently little known paper. Lorentz gave the following necessary and sufficient condition that a set $S$ be uniquely reconstructible. Let the projections $P_{1}(x), P_{2}(y)$ of $S$ onto the $x$-axis and $y$-axis in $\mathbb{R}^{2}$ be

$$
P_{1}(x)=\int \chi_{S}(x, y) d y ; \quad P_{2}(y)=\int \chi_{S}(x, y) d x
$$

and define the nonincreasing rearrangements $p_{i}$ of $P_{i}$ on $0 \leqq u<\infty$ by

$$
\begin{aligned}
p_{i}(u) & =\int \chi\left(x: P_{i}(x) \geqq u\right) d x \\
& =\operatorname{Leb}\left\{x:-\infty<x<\infty \text { and } P_{i}(x) \geqq u\right\},
\end{aligned}
$$

so that $p_{i}(u)$ decreases on $0 \leqq u<\infty$, where Leb $\{\cdot\}$ denotes Lebesgue measure.

THEOREM (Lorentz). A set $S$ in $\mathbb{R}^{2}$ of finite measure is a set of uniqueness if and only if $p_{1}$ and $p_{2}$ are inverses of each other on $u \geqq 0$, i.e.,

$$
p_{1}\left(p_{2}(u)\right) \equiv u, \quad u \geqq 0 .
$$

Lorentz's result can be used to show that the unit disk $D$ is a set of uniqueness. Indeed, it is verified easily that the projections of the unit disk centered at $(0,0)$ are

$$
P_{1}(x)=P_{2}(x)=2 \sqrt{\left(1-x^{2}\right)^{+}}
$$

and that the nonincreasing rearrangements

$$
p_{1}(u)=p_{2}(u)=\sqrt{\left(4-u^{2}\right)^{+}}, \quad u \geqq 0,
$$

are inverses of each other for $u \geqq 0$.

In this paper we give a sufficient condition that $S$ be a set of uniqueness in $\mathbb{R}^{n}$, namely that $S$ be additive (as defined below). We also give a necessary condition that $S$ be a set of uniqueness in $\mathbb{R}^{n}$, which is that $S$ have no bad configuration (as defined below).

A set $S$ is said to be additive if there are bounded measurable functions $f_{i}$ for $i=1, \cdots, n$, where $f_{i}(\mathbf{x})=f_{i}\left(x_{i}\right)$ depends only on the $i$ th coordinate $x_{i}$ of $\mathbf{x}=$ $\left(x_{1}, \cdots, x_{n}\right)$, such that

$$
\mathbf{x} \in S \Leftrightarrow \sum_{i=1}^{n} f_{i}\left(x_{i}\right) \geqq 0
$$

For example, the unit ball is additive as may be seen by taking $f_{i}(\mathbf{x})=(1 / n)-x_{i}^{2}$ for $1 \leqq i \leqq n$.

THEOREM 1.1. Any additive set $S$ in $\mathbb{R}^{n}$ of finite $n$-dimensional measure is a set of uniqueness.

Proof. Suppose there were another set $T$ in $\mathbb{R}^{n}$ having the same projections

$$
P_{i}\left(x_{i}\right)=\iint \cdots \int \chi_{S}(\mathbf{x}) d x_{1} \cdots d x_{i-1} d x_{i+1} \cdots d x_{n}
$$

as $S$. Let $g_{i}(\mathbf{x})=g_{i}\left(x_{i}\right)$ be any integrable (over $S \cup T$ ) function on $\mathbb{R}^{n}$ whose values 
depend only on the $i$ th coordinate of $\mathbf{x}$. Then

$$
\begin{aligned}
\int \ldots_{S} \int g_{i}(\mathbf{x}) d x_{1} \cdots d x_{n} & =\int g_{i}\left(x_{i}\right) P_{i}\left(x_{i}\right) d x_{i} \\
& =\int \cdots \int g_{i}(\mathbf{x}) d x_{1} \cdots d x_{n},
\end{aligned}
$$

by integrating over the $n-1$ coordinates other than $x_{i}$ and using the fact that $S$ and $T$ have the same projections. Clearly, $S$ and $T$ have the same $n$-dimensional measure. Setting $g_{i}=f_{i}$ in (1.3) and subtracting the integral over $S \cap T$ in (1.3) we get

$$
\int \underset{S-S \cap T}{\ldots} \int f_{i}\left(x_{i}\right) d x_{1} \cdots d x_{n}=\int \underset{T-S \cap T}{\ldots} \int f_{i}\left(x_{i}\right) d x_{1} \cdots d x_{n}
$$

and

$$
\int \underset{S-S \cap T}{\cdots} \int d x_{1} \cdots d x_{n}=\int \underset{T-S \cap T}{\ldots} \int d x_{1} \cdots d x_{n} .
$$

But summing over $i$ in (1.4) we obtain

$$
\int \underset{S-S \cap T}{\ldots} \int \sum_{i} f_{i}\left(x_{i}\right) d x_{1} \cdots d x_{n}=\int \underset{T-S \cap T}{\cdots} \int \sum_{i} f_{i}\left(x_{i}\right) d x_{1} \cdots d x_{n} .
$$

The integrand on the left in (1.6) is nonnegative by (1.2), and the integrand on the right in (1.6) is strictly negative, also by (1.2). It follows that Leb $(T-S \cap T)=0$ and then from (1.5) that Leb $(S-S \cap T)=0$, so $S$ and $T$ differ by null sets.

Kemperman [7] has subsequently proved that a generalized notion of our version of additivity also guarantees uniqueness.

We can show that various simple sets are uniquely reconstructible by showing that they are additive, as for the unit $n$-sphere. However, it may be difficult to tell whether a general set $S$ is additive.

There is a simple necessary condition for a set $S$ to be a set of uniqueness, which we state next. A set of four points $\mathbf{z}_{1}=\left(x_{1}, y_{1}\right), \mathbf{z}_{2}=\left(x_{2}, y_{2}\right), \mathbf{w}_{1}=\left(x_{1}, y_{2}\right)$, and $\mathbf{w}_{2}=$ $\left(x_{2}, y_{1}\right)$ forming the corners of a rectangle in $\mathbb{R}^{2}$ are a 2 -bad configuration (or bad rectangle) for $S$ if $\mathbf{z}_{1}$ and $\mathbf{z}_{2}$ are in the interior Int $(S)$ of $S$ and $\mathbf{w}_{1}$ and $\mathbf{w}_{2}$ are in Int $\left(S^{c}\right)$, where $S^{c}=\mathbb{R}^{2}-S$ is the complement of $S$. More generally, a $k$-bad configuration (or $k$-configuration) for $S$ in $\mathbb{R}^{n}$ consists of $2 k$ vectors $\mathbf{z}_{1}, \cdots, \mathbf{z}_{k}$ and $\mathbf{w}_{1}, \cdots, \mathbf{w}_{k}$, where all the $\mathbf{z}_{j}$ are distinct points in Int $(S)$ and all the $\mathbf{w}_{j}$ are distinct points in Int $\left(S^{c}\right)$, and every coordinate plane $x_{i}=c$ contains the same number of $\mathbf{w}_{j}$ 's as $\mathbf{z}_{j}$ 's. We define a weakly $k$-bad configuration for $S$ in $\mathbb{R}^{n}$ the same way, except that $\mathbf{z}_{1}, \cdots, \mathbf{z}_{k}$ need not be distinct points, and $\mathbf{w}_{1}, \cdots, \mathbf{w}_{k}$ need not be distinct points. We say that $S$ has no bad configuration if $S$ has no $k$-bad configuration for any $k \geqq 2$.

THEOREM 1.2. A set $S$ of uniqueness in $\mathbb{R}^{n}$ has no bad configuration.

Proof. Suppose $S$ has a $k$-bad configuration consisting of points $\mathbf{z}_{1}, \cdots, \mathbf{z}_{k}$ and $\mathbf{w}_{1}, \cdots, \mathbf{w}_{k}$ as described above. Then for a small enough positive $\delta$, the set $C_{1}$ consisting of the union of $k$ balls of radius $\delta$ around the $\mathbf{z}_{i}$ lies in Int $(S)$, and the set $C_{2}$ consisting of the union of $k$ balls of radius $\delta$ around the $\mathrm{w}_{i}$ lies in Int $\left(S^{c}\right)$. Then $T=S-C_{1}+C_{2}$ has the same projections as $S$ so $S$ cannot be a set of uniqueness.

In the rest of this paper we study the relations between the conditions that a set $S$ is additive, that $S$ is a set of uniqueness, and that $S$ has no bad configuration. We now summarize the results obtained.

In $\S 2$ we first show that the concepts "having a bad configuration" and "having a weakly bad congfiuration" coincide for open sets in all dimensions. 
THEOREM 2.1. Let $S$ be an open set of finite measure in $\mathbb{R}^{n}$. The following are equivalent.

(1) $S$ has no $k$-bad configuration for any $k \geqq 2$.

(2) $S$ has no weakly $k$-bad configuration for any $k \geqq 2$.

This theorem highlights an essential difference between the continuous case and the discrete case, for in [1] we show that in the discrete case the concepts "having a bad configuration" and "having a weakly bad configuration" are distinct in all dimensions greater than or equal to 3 .

In $\S 2$ we study the two-dimensional case in detail. In the two-dimensional case, additivity and uniqueness coincide.

THEOREM 2.2. Let $S$ be a set in $\mathbb{R}^{2}$ of finite measure. The following conditions are equivalent.

(1) $S$ is a set of uniqueness.

(2) $S$ is additive.

Kuba and Volčič [8] give other necessary and sufficient conditions for being a set of uniqueness in the two-dimensional case.

If $S$ is suitably restricted then the "set of uniqueness" concept essentially coincides with the other concepts in the two-dimensional case.

THEOREM 2.3. Let $S$ be an open set in $\mathbb{R}^{2}$ of finite measure. The following conditions are equivalent.

(1) There is no open set $\tilde{S}$ unequal to $S$ having the same projections on the $x$-axis and $y$-axis.

(2) $S$ has no $k$-bad configuration for all $k \geqq 2$.

(3) $S$ has no weakly $k$-bad configuration for all $k \geqq 2$.

If in addition the boundary $\partial S$ has measure zero, these are also equivalent to the following condition.

(4) S has no 2-bad configuration.

It seems likely that the hypothesis that $\partial S$ has zero measure is unnecessary in this theorem.

An interesting question concerns whether or not the concepts of uniqueness and additivity coincide for three or more dimensions. In an earlier version of this paper we conjectured that they coincide, but Kemperman [6] settled that conjecture with a counterexample of a set of uniqueness that is not additive. Kellerer found a similar counterexample and kindly transmitted it to us. Kemperman [7] then proposed a generalized notion of additivity that is necessary and sufficient for certain sets to be sets of uniqueness.

Section 3 studies general properties of additive sets. We first give a criterion for a set not to be additive.

THEOREM 3.1. If $S$ is a bounded measurable subset of $\mathbb{R}^{n}$ and if there are measures $\mu$ and $\nu$ on $\mathbb{R}^{n}$ with the same projections (one-dimensional marginals) on all $n$ axes, and $\mu$ is concentrated on $S$ and $\nu$ is concentrated on $S^{c}$, then $S$ is not additive.

Section 3 then studies the following subset of the cube in $\mathbb{R}^{3}$ :

$$
S_{0}=\left\{\left(x_{1}, x_{2}, x_{3}\right): 0 \leqq x_{i} \leqq 1, i=1,2,3, x_{3} \geqq \max \left\{x_{1}, x_{2}\right\}\right\} \text {. }
$$

Note that $S_{0}$ is the limit as $n \rightarrow \infty$ of the additive sets

$$
S_{n}=\left\{\left(x_{1}, x_{2}, x_{3}\right): 0 \leqq x_{i} \leqq 1 \text { for } i=1,2,3, x_{3}^{n} \geqq x_{1}^{n}+x_{2}^{n}\right\},
$$

with $S_{1} \subset S_{2} \subset S_{3} \subset \cdots$. Our study of this set was motivated by our search for a set of uniqueness that is not additive. We prove two interesting facts about $S_{0}$.

THEOREM 3.2. $S_{0}$ is not additive. 
THEOREM 3.3. $S_{0}$ is not a set of uniqueness.

These theorems imply that both the additivity property and the set of uniqueness property are not closed under the operation of taking monotone pointwise limits in dimensions greater than or equal to 3 .

Theorem 3.2 follows from the fact that there are a pair of measures $\mu$ and $\nu$ concentrated on $S_{0}$ and $S_{0}^{c}$, respectively, having the same projections onto the coordinate axes. An alternative direct proof of Theorem 3.2 has been shown to us by Kemperman.

We prove Theorem 3.3 by showing there exists a pair of measures $\mu$ and $\nu$ concentrated on $S_{0}$ and $S_{0}^{c}$, respectively, having densities taking only the values 0 and 1 , which have the same projections on the coordinate axes. We actually construct a pair $\mu, \nu$ having bounded densities and then use a general result ([2]) which asserts that the existence of a pair $(\mu, \nu)$ with bounded densities implies the existence of such a pair having densities taking only the values 0 and 1 .

The related problem of deciding whether there exists a set $S$ having given projections is not considered in the paper. The existence problem is studied in the twodimensional continuous case in [5], [9], where necessary and sufficient conditions for existence are given.

2. Continuous case: general results. Our first result in the continuous case shows that the concepts of "no bad configuration" and "no weakly bad configuration" coincide for open sets $S$, in all dimensions $n$. This differs from the discrete case for $n \geqq 3$.

THEOREM 2.1. Let $S$ be an open set in $\mathbb{R}^{n}$. The following conditions are equivalent.

(1) $S$ has no weakly $k$-bad configuration for all $k \geqq 2$.

(2) $S$ has no $k$-bad configuration for all $k \geqq 2$.

Proof. It is clear that $(2) \Rightarrow(1)$, so that it suffices to show $(1) \Rightarrow(2)$. To do this we show that if $S$ has a weakly $k$-bad configuration then it has a $k$-bad configuration. Let the weakly $k$-bad configuration consist of the $k$ points $\mathbf{w}^{(i)}=\left(w_{1}^{(i)}, \cdots, w_{n}^{(i)}\right)$ in $\operatorname{Int}(S)$, and the $k$ points $\mathbf{z}^{(i)}=\left(z_{1}^{(i)}, \cdots, z_{n}^{(i)}\right)$ in Int $\left(S^{c}\right)$, for $1 \leqq i \leqq k$. Since the sets $\left\{w_{j}^{(i)}\right\}$ and $\left\{z_{j}^{(i)}\right\}$ have equal projections (counting multiplicities) for each $j$ from 1 to $n$, there are permutations $\pi_{j}$ of $\{1,2, \cdots, k\}$ such that $z_{j}^{(i)}=w_{j}^{\left(\pi_{j}(i)\right)}$. Clearly, there are $k$ points $\hat{\mathbf{w}}^{(i)}$ arbitrarily close to the $\mathbf{w}^{(i)}$ in Int $(S)$ whose coordinates consist of $k n$ distinct real numbers. For any such $\hat{\mathbf{w}}^{(i)}$, define $\hat{\mathbf{z}}^{(i)}$ by $\hat{z}_{j}^{(i)}=\hat{w}_{j}^{\left(\pi_{j}^{(i))}\right.}$ for all $i$ and $j$. Then the $\hat{\mathbf{z}}^{(i)}$ and $\hat{\mathbf{w}}^{(i)}$ are all distinct points and the sets $\left\{\hat{\mathbf{w}}^{(i)}\right\}$ and $\left\{\hat{\mathbf{z}}^{(i)}\right\}$ have the same projections. As $\hat{\mathbf{w}}^{(i)}$ approaches $\mathbf{w}^{(i)}, \hat{\mathbf{z}}^{(i)}$ approaches $\mathbf{z}^{(i)}$, so by choosing the $\hat{\mathbf{w}}^{(i)}$ sufficiently close to the $\mathbf{w}^{(i)}$ we may ensure both $\hat{\mathbf{w}}^{(i)} \in \operatorname{Int}(S)$ and $\hat{\mathbf{z}}^{(i)} \in \operatorname{Int}\left(S^{c}\right)$; hence we conclude that there is a $k$-bad configuration.

The remainder of this section establishes results for the two-dimensional case.

THEOREM 2.2. Let $S$ be a set of finite measure in $\mathbb{R}^{2}$. The following are equivalent.

(1) $S$ is a set of uniqueness.

(2) $S$ is additive.

Proof. The result $(2) \Rightarrow(1)$ is just Theorem 1.1. To prove $(1) \Rightarrow(2)$ we use ideas of Lorentz [9]. We are given a set of uniqueness $S$. Suppose first that $S$ is contained in $\mathbb{R}_{+}^{2}=\left\{\left(x_{1}, x_{2}\right): x_{1} \geqq 0, x_{2} \geqq 0\right\}$ and that the projection

$$
P_{1}\left(x_{1}\right)=\int_{-\infty}^{\infty} \chi_{S}\left(x_{1}, x_{2}\right) d x_{2}
$$

is nonincreasing for $x_{1} \geqq 0$, and that the projection

$$
P_{2}\left(x_{2}\right)=\int_{-\infty}^{\infty} \chi_{S}\left(x_{1}, x_{2}\right) d x_{1}
$$


is nonincreasing for $x_{2} \geqq 0$. Note that $P_{1}\left(x_{1}\right)$ is finite for each $x_{1}>0$ since $S$ has finite measure. Lorentz's condition that $S$ be a set of uniqueness is

$$
P_{2}\left(P_{1}\left(x_{1}\right)\right) \equiv x_{1} \quad \text { a.e. [Leb] }
$$

for $x_{1} \geqq 0$. Using this it is easy to verify that

$$
S=\left\{\left(x_{1}, x_{2}\right): x_{1} \geqq 0 \text { and } 0 \leqq x_{2} \leqq P_{1}\left(x_{1}\right)\right\}
$$

is identical to $S$ up to a null set, since the right side of (2.1) produces the correct projections. Using the fact that $P_{1}\left(x_{1}\right)$ is nonincreasing, it is easy to verify that

$$
S^{\prime}=\left\{\left(x_{1}, x_{2}\right): f_{1}\left(x_{1}\right)+f_{2}\left(x_{2}\right) \geqq 0\right\}
$$

where

$$
f_{1}\left(x_{1}\right)= \begin{cases}P_{1}\left(x_{1}\right) & \text { if } x_{1} \geqq 0 \\ -\infty & \text { if } x_{1}<0\end{cases}
$$

and

$$
f_{2}\left(x_{2}\right)= \begin{cases}-x_{2} & \text { if } x_{2} \geqq 0 \\ -\infty & \text { if } x_{2}<0 .\end{cases}
$$

This would show that $S$ is additive if $f_{1}$ and $f_{2}$ did not take the value $-\infty$. We avoid this difficulty in the general case by a change of variable, as described below.

Consider the general case and recall that Lorentz proves that associated to each projection $P_{i}\left(x_{i} ; S\right)$ there are measurable functions $\sigma_{i}: \mathbb{R} \rightarrow \mathbb{R}^{+}=\{x: x>0\}$ which are one-one (almost everywhere) and onto (almost everywhere) such that the functions $p_{i}(t)$ defined by

$$
p_{i}\left(\sigma_{i}\left(x_{i}\right)\right)=P_{i}\left(x_{i}\right) \quad i=1,2
$$

are nonincreasing on $0<t<\infty$. These are monotone rearrangements of the $P_{i}\left(x_{i}\right)$ on the $x_{i}$-coordinates. Now define $S^{+} \subseteq \mathbb{R}^{+} \times \mathbb{R}^{+}$by

$$
S^{+}=\left\{\left(\sigma_{1}\left(x_{1}\right), \sigma_{2}\left(x_{2}\right)\right):\left(x_{1}, x_{2}\right) \in S\right\} .
$$

Then $S^{+}$has projections $p_{1}\left(x_{1}\right)$ and $p_{2}\left(x_{2}\right)$, and it is easy to check that $S^{+}$is a set of uniqueness if and only if $S$ is a set of uniqueness. By hypothesis, $S$ is a set of uniqueness, hence so is $S^{+}$, and by the preceding argument there are functions $f_{1}$ and $f_{2}$ for $S^{+}$ satisfying (2.2)-(2.4). Then (2.2) implies that $S$ is additive for $f_{1}^{*}\left(x_{1}\right)$ and $f_{2}^{*}\left(x_{2}\right)$, where

$$
f_{1}^{*}\left(x_{1}\right)=f_{1}\left(\sigma_{1}\left(x_{1}\right)\right) \text { and } f_{2}^{*}\left(x_{2}\right)=f_{2}\left(\sigma_{2}\left(x_{2}\right)\right)
$$

after noting that $f_{1}^{*}\left(x_{1}\right)$ and $f_{2}^{*}\left(x_{2}\right)$ are finite everywhere because $\sigma_{1}\left(x_{1}\right)>0$ and $\sigma_{2}\left(x_{2}\right)>0$ for all $x_{1}, x_{2} \in \mathbb{R}$. This completes the proof.

We now observe that for open sets $S$ of finite measure our concepts essentially coincide in the two-dimensional case.

THEOREM 2.3. Let $S$ be an open set in $\mathbb{R}^{2}$ of finite measure. The following conditions are equivalent.

(1) There is no open set $\tilde{S}$ unequal to $S$ having the same projections on the $x$-axis and $y$-axis.

(2) $S$ has no $k$-bad configuration for all $k \geqq 2$.

(3) $S$ has no weakly $k$-bad configuration for all $k \geqq 2$.

If in addition the boundary $\partial S$ of $S$ has measure zero, then these conditions are also equivalent to the following one.

(4) S has no 2-bad configuration.

Proof. Condition $(1) \Rightarrow(2)$ by Theorem 1.2 and $(2) \Leftrightarrow(3)$ by Theorem 2.1. We prove $(2) \Rightarrow(1)$ in the contrapositive form by the following lemma. 
Lemma 2.4. Suppose that $S_{1}$ and $S_{2}$ are disjoint open sets in $\mathbb{R}^{2}$ of finite area having the same projections on the $x$-axis and $y$-axis. Then there are finite sets $T_{1}$ in $S_{1}$ and $T_{2}$ in $S_{2}$ having the same number of points on each line $x=c$ and on each line $y=c$ for all $c \in \mathbb{R}$.

Proof. We prove the stronger result that the set $T_{1}$ can include any given point $\mathbf{v}_{0}=\left(x_{0}, y_{0}\right)$ in $S_{1}$. To do this take a small open square $U_{0}$ of side $\delta$ centered at $\mathbf{v}_{0}$ that lies entirely in the open set $S_{1}$. Since the projections of $S_{1}$ and $S_{2}$ agree, we can find points $\mathbf{y}_{1}=\left(x_{0}, y_{1}\right)$ and $\mathbf{y}_{2}=\left(x_{1}, y_{0}\right)$ with $\mathbf{y}_{1}$ and $\mathbf{y}_{2}$ in $S_{2}$. Since $S_{2}$ is open, by decreasing $\delta$ if necessary, we can find open squares $U_{0}, U_{1}^{*}, U_{1}$ of side $\delta$ centered at $\mathbf{v}_{0}, \mathbf{y}_{1}$, and $\mathbf{y}_{2}$ with the squares $U_{1}, U_{1}^{*}$ being in $S_{2}$. (See Fig. 2.1.) We say that the points in $U_{1}^{*}$ cover those in $U_{0}$ in the "down" direction, by which we mean the projections $P_{1}\left(x ; U_{1}^{*}\right)=P_{1}\left(x ; U_{0}\right)$ for all $x \in \mathbb{R}$, and those in $U_{1}$ cover those in $U_{0}$ in the "across" direction, by which we mean $P_{2}\left(y ; U_{1}\right) \equiv P_{2}\left(y ; U_{0}\right)$. Note that $U_{0}, U_{1}$, and $U_{1}^{*}$ each has area $\delta^{2}$.
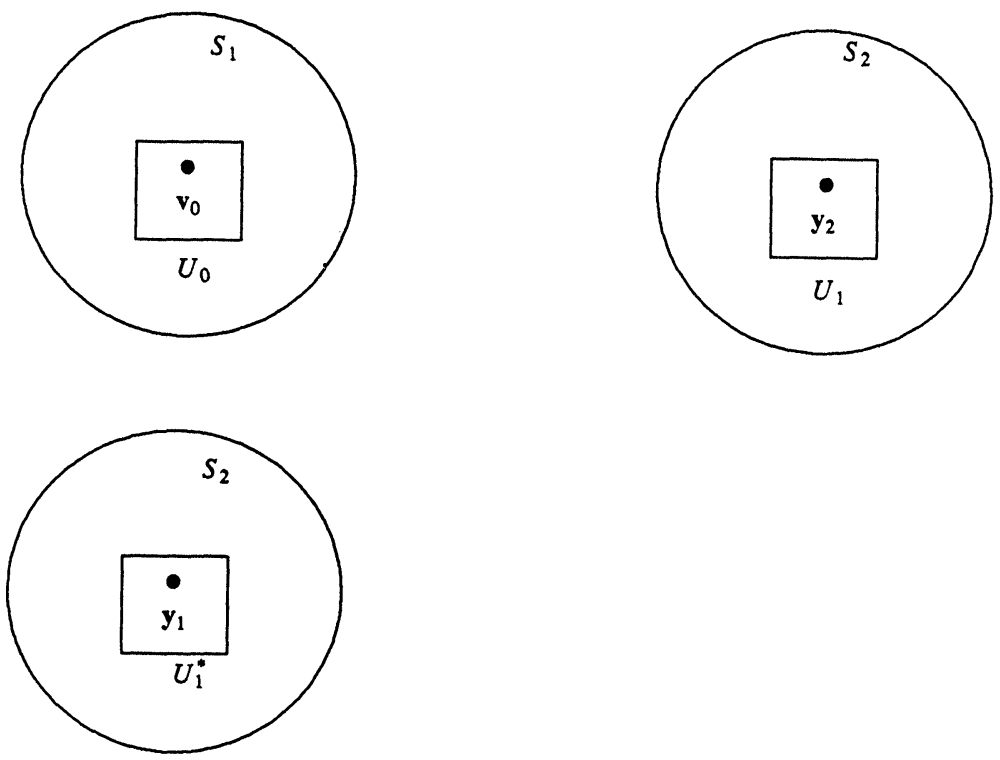

FIG. 2.1. Initial open squares in $S_{1}$ and $S_{2}$.

Now let $\varepsilon$ be a positive constant depending on $\delta$ that will be picked once and for all. We claim there exists an open set $U_{2}$ such that:

(i) $U_{2}$ is in $S_{1}$.

(ii) For each point $\mathbf{w}=\left(w_{1}, w_{2}\right)$ in $U_{2}$ there is some point $\left(w_{1}, y\right)$ in $U_{1}$ having the same first coordinate.

(iii) $P_{1}\left(x ; U_{2}\right) \leqq P_{1}\left(x ; U_{1}\right)$ for all $x$.

(iv) $\int_{-\infty}^{\infty}\left|P_{1}\left(x ; U_{2}\right)-P_{1}\left(x ; U_{1}\right)\right| d x<\varepsilon$, so that Area $\left(U_{2}\right) \geqq \delta^{2}-\varepsilon$.

(v) $U_{2}$ is a disjoint union of a finite set of open rectangles.

To see this, we use the fact that since $U_{1}$ is in $S_{2}$, and $S_{1}$ and $S_{2}$ have the same projections, and since $U_{0}$ has no $x$-coordinate in common with $U_{1}$, then

$$
P_{1}\left(x ; S_{1}-\bar{U}_{0}\right) \geqq P_{1}\left(x ; U_{1}\right) \text { for all } x \in \mathbb{R},
$$

where the bar denotes closure. Now cover the open set $S_{1}-\bar{U}_{0}$ with a mesh of squares of side $\eta$ and let $\eta \rightarrow 0$. Taking $\eta$ sufficiently small we can approximate the Riemann 
integral of $S_{1}-\bar{U}_{0}$ arbitrarily well from below, and choosing an appropriate subcollection of these squares yields a set $U_{2}$ such that (i)-(v) hold.

We continue to construct a series of sets $U_{i}$ such that:

(i) If $i$ is odd, then $U_{i}$ is in $S_{2}$, and is in $S_{1}$ otherwise.

(ii) Each point $w=\left(w_{1}, w_{2}\right)$ in $U_{i}$ can be reached from a point $\left(w_{1}, y\right)$ in $U_{i-1}$ if $i$ is even and from a point $\left(x, w_{2}\right)$ in $U_{i-1}$ if $i$ is odd.

(iii) $P_{1}\left(x ; U_{i}\right) \leqq P_{1}\left(x ; U_{i-1}\right)$ for all $x$ if $i$ is even. $P_{2}\left(y ; U_{i}\right) \leqq P_{2}\left(y ; U_{i-1}\right)$ for all $y$ if $i$ is odd.

(iv) $\int_{-\infty}^{\infty}\left|P_{j}\left(z ; U_{i}\right)-P_{j}\left(z ; U_{i-1}\right)\right| d z \leqq i \varepsilon$ where $j=1$ for even $i$ and $j=2$ for odd $i$, so Area $\left(U_{i}\right) \geqq \delta^{2}-i^{2} \varepsilon$.

(v) $U_{i}$ is a disjoint union of a finite set of open rectangles.

(vi) $U_{i} \cap U_{j}=\varnothing$ for $0 \leqq j<i$.

This construction is carried out using the fact that (for $i=2 j$ )

$$
P_{1}\left(x ; S_{1}-\bar{U}_{0}-\bar{U}_{2}-\cdots-\bar{U}_{2 j-2}\right) \geqq P_{1}\left(x ; U_{2 j-1}\right)
$$

and (for $i=2 j+1)$

$$
P_{2}\left(y ; S_{2}-\bar{U}_{1}-\bar{U}_{3}-\cdots-\bar{U}_{2 j-1}\right) \geqq P_{2}\left(y ; U_{2 j}\right)
$$

provided $U_{2 j+1} \cap U_{1}^{*}=\varnothing$. Since $S_{1}$ has finite area $A$, if we take $\varepsilon$ small enough, say $\varepsilon=1 / 100 A^{-2} \delta^{4}$, then the sets $U_{i}$ are all of area greater than $\frac{1}{2} \delta^{2}$ for $1 \leqq i \leqq 2(A / \delta)$, hence by pairwise disjointness this construction must halt for some $i<2(A / \delta)$. It follows that there is some $i=2 j$ for which $U_{2 j}$ has a point $\mathbf{v}_{2 j}=\left(x_{2 j}, y_{2 j}\right)$ such that there is a point $\mathbf{v}=\left(x^{*}, y_{2 j}\right)$ in $U_{1}^{*}$. By condition (ii) we can extend this to a sequence of points $\mathbf{v}_{k}=\left(x_{k}, y_{k}\right)$ in $U_{k}$ such that $x_{k}=x_{k+1}$ if $k$ is odd and $y_{k}=y_{k+1}$ if $k$ is even. Now since $U_{1}^{*}$ and $U_{1}$ are rectangles, $\mathbf{v}_{1}=\left(x_{1}, y_{0}\right) \in U_{1}$ and $\mathbf{v}_{2 j+1}=\left(x_{0}, y_{2 j}\right) \in U_{1}^{*}$. Then the sets of points $T_{1}=\left\{\mathbf{v}_{0}, \mathbf{v}_{2}, \mathbf{v}_{4}, \cdots, \mathbf{v}_{2 j}\right\}$ in $S_{1}$ and $T_{2}=\left\{\mathbf{v}_{1}, \mathbf{v}_{3}, \mathbf{v}_{5}, \cdots, \mathbf{v}_{2 j+1}\right\}$ in $S_{2}$ have the required property.

We now complete the proof of Theorem 2.3. The implication $(2) \Rightarrow(4)$ is always true. We prove $(4) \Rightarrow(2)$ under the assumption that the boundary $\partial S=\bar{S}-S$ of $S$ has Lebesgue measure zero. (Since open sets are Lebesgue measurable, it follows that $\partial S$ is always measurable, but it may have positive measure; cf. [12, p. 59].) We show the contrapositive, which asserts that if $S$ has a $k$-bad configuration for $k \geqq 3$ then $S$ has a 2-bad configuration.

We may suppose that the $k$-bad configuration has the form

$$
\begin{aligned}
& \mathbf{x}^{(1)}=\left(x_{1}^{(1)}, y_{1}^{(1)}\right) \in \operatorname{Int}(S), \quad \mathbf{x}^{(2)}=\left(x_{1}^{(1)}, y_{1}^{(2)}\right) \in \operatorname{Int}\left(S^{c}\right), \\
& \mathbf{x}^{(3)}=\left(x_{1}^{(2)}, y_{1}^{(2)}\right) \in \operatorname{Int}(S), \quad \mathbf{x}^{(4)}=\left(x_{1}^{(2)}, y_{1}^{(3)}\right) \in \operatorname{Int}\left(S^{c}\right), \\
& \mathbf{x}^{(2 k-1)}=\left(x_{1}^{(k)}, y_{1}^{(k)}\right) \in \operatorname{Int}(S), \quad \mathbf{x}^{(2 k)}=\left(x_{1}^{(k)}, y_{1}^{(1)}\right) \in \operatorname{Int}\left(S^{c}\right) .
\end{aligned}
$$

Since these sets are open, for all $\mathbf{y}$ with $\|\mathbf{y}\|<\delta$ and $\delta$ small enough, the set

$$
S(\mathbf{y})=\left\{\mathbf{x}^{(i)}+\mathbf{y}: 1 \leqq i \leqq 2 k\right\}
$$

is a $k$-bad configuration. Since $\partial S$ has measure 0 , the set of $\mathbf{y}$ with $\|\mathbf{y}\|<\delta$ for which some point $\mathbf{x}^{(i)}+\mathbf{y}$ is in $\partial S$ has measure 0 , hence we can choose $\mathbf{y}$ so that all $k^{2}$ points $\left(x_{1}^{(i)}, y_{1}^{(j)}\right)$ for $1 \leqq i, j \leqq k$ are in $\operatorname{Int}(S) \cup \operatorname{Int}\left(S^{c}\right)$, i.e., none of them are on $\partial S\left(=\partial S^{c}\right)$. Now the argument of Lemma 1 in [1] in the discrete case can be applied to conclude that there is a subset of four of these $k^{2}$ points with $\left\{\left(x_{1}, y_{1}\right),\left(x_{2}, y_{2}\right)\right\} \subseteq S$ and $\left\{\left(x_{1}, y_{2}\right),\left(x_{2}, y_{1}\right)\right\} \subseteq S^{c}$. Since these points are actually in $\operatorname{Int}(S)$ or $\operatorname{Int}\left(S^{c}\right)$, this is a 2-bad configuration. 


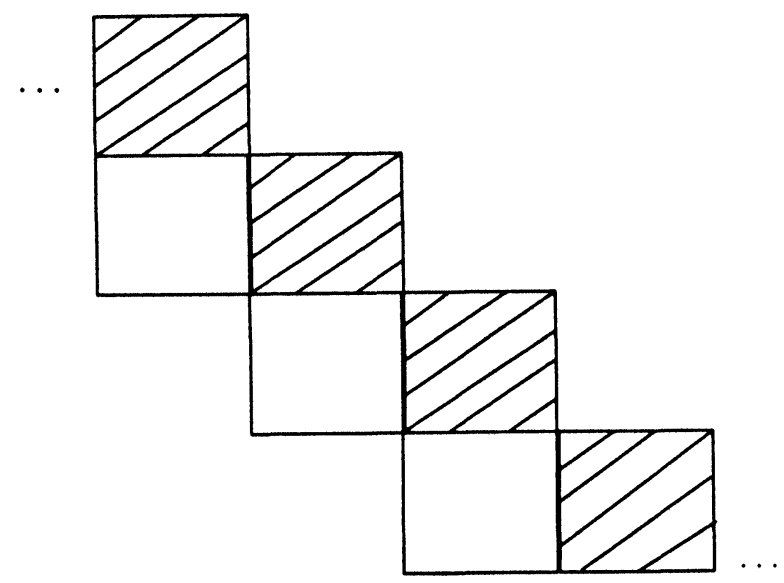

FIG. 2.2. Staircase example.

Remarks. (1) Lemma 2.4 is false in two-dimensions if $S_{1}$ and $S_{2}$ are allowed to have infinite area, even if projections are everywhere finite. The infinite checkerboard staircase in Fig. 2.2 provides a counterexample, where shaded open squares are in $S_{1}$ and unshaded open squares are in $S_{2}$.

(2) The three-dimensional analogue of Lemma 2.4 is false for open sets $S_{1}$ and $S_{2}$ of finite volume.

Lemma 2.5. There exist two disjoint bounded open sets $S_{1}$ and $S_{2}$ in $\mathbb{R}^{3}$ such that:

(1) $S_{1}$ and $S_{2}$ have identical projections on all three axes.

(2) There exists no finite bad configuration for $S_{1}$ or $S_{2}$ contained in $S_{1} \cup S_{2}$.

This result is proved in the Appendix.

3. Properties of additive sets. Recall that $S$ is additive if and only if there are bounded measurable functions $f_{i}\left(x_{i}\right)$ for $1 \leqq i \leqq n$ such that, up to sets of measure zero,

$$
S=\left\{\mathbf{x}=\left(x_{1}, \cdots, x_{n}\right): \sum_{i=1}^{n} f_{i}\left(x_{i}\right) \geqq 0\right\} .
$$

Although it is difficult to decide when a set is additive, there is a simple necessary condition for additivity.

THEOREM 3.1. Suppose $S$ is a bounded measurable subset of $\mathbb{R}^{n}$ and there are measures $\mu$ and $\nu$ with the same projections for which $\mu$ and $\nu$ are concentrated on $S$ and $S^{c}$, i.e., $\mu(S)>0$ and

$$
\mu\left(S^{c}\right)=\nu(S)=0 .
$$

Then $S$ is not additive, i.e., (3.1) holds for no $f_{i}, i=1, \cdots, n$.

Proof. If $f_{i}$ exist satisfying (3.1) then for each $i$

$$
\int \cdots \int f_{i}\left(x_{i}\right) d \mu(x)=\int \cdots \int f_{i}\left(x_{i}\right) d \nu(x),
$$

since the $f_{i}$ are bounded measurable and depend only on one coordinate while $\mu$ and $\nu$ have the same one-dimensional margins. Adding over $i$ in (3.3) gives

$$
\int \ldots \int \sum_{i=1}^{n} f_{i}\left(x_{i}\right) d \mu(x)=\int \ldots \sum_{S^{c}}^{n} \sum_{i=1}^{n} f_{i}\left(x_{i}\right) d \nu(x) .
$$

By (3.1), the left integrand is nonnegative while the right integrand is strictly negative. 
It follows that $\nu$ has measure zero and so $\mu$ has measure zero since $\mu$ and $\nu$ have the same marginals. But $\mu(S)>0$, so $f_{i}$ cannot exist.

To illustrate some of the difficulties in proving that a set is additive or is one of uniqueness, we study the following particular subset $S_{0}$ of the cube in $\mathbb{R}^{3}$ :

$$
S_{0}=\left\{\left(x_{1}, x_{2}, x_{3}\right): x_{3} \geqq \max \left\{x_{1}, x_{2}\right\}, 0 \leqq x_{i} \leqq 1 \text { for } i=1,2,3\right\} .
$$

We will show that $S_{0}$ is not additive by exhibiting a pair of measures satisfying Theorem 3.1 for $S_{0}$.

TheOREM 3.2. $S_{0}$ is not additive, i.e., there are $\mu$ and $\nu$ satisfying (3.2) with $S=S_{0}$.

Proof. Since we would naturally believe that $\max \left\{x_{1}, x_{2}\right\}$ cannot be written as $f_{3}^{-1}\left(f_{1}\left(x_{1}\right)+f_{2}\left(x_{2}\right)\right)$, the result that $S_{0}$ is not additive is perhaps not surprising.

The proof gives a $\mu$ and $\nu$ not absolutely continuous with respect to Lebesgue measure $\lambda$. The idea behind the proof is that if we can find a single probability space on which random variables $X_{1}, X_{2}, X_{1}^{\prime}, X_{2}^{\prime}$ are defined, and if $X_{i}$ and $X_{i}^{\prime}$ have the same distribution for $i=1,2$, and yet $\max \left\{X_{1}, X_{2}\right\}$ is everywhere strictly less than $\max \left\{X_{1}^{\prime}, X_{2}^{\prime}\right\}$, i.e.,

$$
X_{1} \sim X_{1}^{\prime}, \quad X_{2} \sim X_{2}^{\prime}, \quad \max \left\{X_{1}, X_{2}\right\}<\max \left\{X_{1}^{\prime}, X_{2}^{\prime}\right\},
$$

then we may assume by change of scale that $0 \leqq X_{i} \leqq 1$ for $i=1,2$ and set

$$
X_{3}=X_{3}^{\prime}=\frac{1}{2}\left(\max \left\{X_{1}, X_{2}\right\}+\max \left\{X_{1}^{\prime}, X_{2}^{\prime}\right\}\right) \text {. }
$$

Then the measure $\mu$ induced by $\left(X_{1}, X_{2}, X_{3}\right)$ is concentrated on $S_{0}$ since $X_{3} \geqq$ $\max \left\{X_{1}, X_{2}\right\}$ while the measure $\nu$ induced by $\left(X_{1}^{\prime}, X_{2}^{\prime}, X_{3}^{\prime}\right)$ is concentrated on $S_{0}^{c}$ since $X_{3}^{\prime}<\max \left\{X_{1}^{\prime}, X_{2}^{\prime}\right\}$. The measures $\mu$ and $\nu$ have the same projections onto the first two coordinate axes since $X_{i} \sim X_{i}^{\prime}$ for $i=1,2$; they also have the same projections onto $x_{3}$ since $X_{3}=X_{3}^{\prime}$.

We construct random variables satisfying (3.4). Let $X_{1}=X_{2}=\beta$ where

$$
P(\beta \in d u)=\frac{d u}{\pi \sqrt{u(1-u)}}, \quad 0 \leqq u \leqq 1,
$$

and let $\left(X_{1}^{\prime}, X_{2}^{\prime}\right)$ be a uniform point on the union of the line segments from $(0,1)$ to $(\beta, \beta)$ and from $(\beta, \beta)$ to $(1,0)$. Clearly $\max \left\{X_{1}, X_{2}\right\}=\beta<\max \left\{X_{1}^{\prime}, X_{2}^{\prime}\right\}$. It remains only to show that $X_{1}^{\prime}$ and $X_{2}^{\prime}$ have the distribution of $\beta$. We have

$$
\begin{aligned}
P\left(X_{1}^{\prime} \in d u\right) & =\int_{0}^{1} P(\beta \in d \nu) P\left(X_{1}^{\prime} \in d u \mid \beta \in d \nu\right) \\
& =\int_{0}^{u} \frac{d \nu}{\pi \sqrt{\nu(1-\nu)}} \frac{d u}{2(1-\nu)}+\int_{u}^{1} \frac{d \nu}{\pi \sqrt{\nu(1-\nu)}} \frac{d u}{2 \nu}=\frac{d u}{\pi \sqrt{u(1-u)}} .
\end{aligned}
$$

Hence $X_{1}^{\prime} \sim \beta$ and therefore $X_{2}^{\prime} \sim X_{1}^{\prime}$. Of course since $\mu$ assigns measure 1 to $X_{1}=X_{2}$, $\mu$ is not absolutely continuous.

We now show that $S_{0}$ is not a set of uniqueness, hence that neither additivity nor being a set of uniqueness is closed under monotone pointwise limits for $n$ greater than or equal to 3 . To do this it suffices to show that there are measures $\mu$ and $\nu$ satisfying the conditions of Theorem 3.1 for $S_{0}$ which have Radon-Nikodym derivative 0 and 1 at all points. Indeed, if $T_{0}$ is another set with the same projections as $S_{0}$ and if $\lambda=$ Lebesgue measure on $\mathbb{R}^{n}, \chi(A)$ is the indicator function of $A$, and $\mu_{0}$ and $\nu_{0}$ are the measures with Radon-Nikodym derivatives

$$
\frac{d \mu_{0}}{d \lambda}=\chi\left(S_{0}-S_{0} \cap T_{0}\right) \quad \text { and } \quad \frac{d \nu_{0}}{d \lambda}=\chi\left(T_{0}-S_{0} \cap T_{0}\right)
$$

then $\mu_{0}$ and $\nu_{0}$ have the same projections. 
THEOREM 3.3. $S_{0}$ is not a set of uniqueness, i.e., there are $\mu$ and $\nu$ with densities taking only values 0 and 1 satisfying $\mu\left(S_{0}^{c}\right)=\nu\left(S_{0}\right)=0$.

Proof. The idea of the proof is to construct $\mu$ and $\nu$ with bounded densities satisfying (3.2), and then to use a general result that the existence of $\mu$ and $\nu$ with bounded densities implies the existence of such $\mu$ and $\nu$ having densities taking only values 0 and 1 .

We now exhibit $\mu$ and $\nu$ with bounded densities that satisfy (3.2). We will construct a probability space and random variables as in (3.4). To this end suppose $q(y)>0$ on $I=(0,1)$ and define the measure $\pi$ on $I^{2}$ by

$$
\pi(d x, d y)=q(x \vee y) d x d y, \quad \text { where } x \vee y=\max \{x, y\} .
$$

We will define a mapping $(x, y) \rightarrow\left(x^{\prime}, y^{\prime}\right)$ with $x \vee y<x^{\prime} \vee y^{\prime}$ and then set $X_{1}=x, X_{2}=y$, $X_{1}^{\prime}=x^{\prime}, X_{2}^{\prime}=y^{\prime}$ so that (3.4) holds. To do this, we choose $\phi(y): I \rightarrow I$ and $\psi(y): I \rightarrow I$ and set

$$
\begin{aligned}
& y^{\prime}=\phi(y), \quad x^{\prime}=\frac{x}{y} \psi(y) \quad \text { for } x<y \\
& x^{\prime}=\phi(x), \quad y^{\prime}=\frac{y}{x} \psi(x) \quad \text { for } y<x
\end{aligned}
$$

where $\phi$ and $\psi$ are to satisfy

$$
y<\phi(y), \quad \phi(0)=0, \quad \phi(1)=1, \quad \phi \text { increases, } \quad 0<\psi(y)<1 .
$$

For (3.4) to hold we need $\pi(x<a) \equiv \pi\left(x^{\prime}<a\right)$ so that we want

$$
\begin{aligned}
\int_{0}^{a} d x \int_{0}^{x} d y q(x)+\int_{0}^{a} d x \int_{x}^{1} d y q(y)= & \int_{0}^{1} d x \int_{0}^{x} d y q(x) \chi(\phi(x)<a) \\
& +\int_{0}^{1} d x \int_{x}^{1} d y q(y) \chi\left(\frac{x}{y} \psi(y)<a\right) .
\end{aligned}
$$

We will require $\psi$ and $q$ to satisfy

$$
\psi(y) \equiv \alpha \quad \text { where } 0<\alpha<1 \text { is to be chosen }
$$

and

$$
\begin{gathered}
a q(a)>\int_{0}^{a} q(y) d y, \quad 0<a<\alpha \\
\alpha \int_{0}^{1} q(y) d y=\int_{0}^{1} y q(y) d y, \quad q(a)>0, \quad 0<a<1 .
\end{gathered}
$$

Then (3.8) with $b=b(a)=\phi^{-1}(a)$ may be written as

$$
2 \int_{0}^{a} x q(x) d x+a \int_{a}^{1} q(y) d y=\int_{0}^{b} x q(x) d x+\min \left\{\frac{a}{\alpha}, 1\right\} \int_{0}^{1} y q(y) d y .
$$

Clearly $b(0)=0, b(1)=1$, and differentiating on $a$ we obtain

$$
\begin{gathered}
a q(a)+\int_{a}^{1} q(y) d y-\frac{1}{\alpha} \int_{0}^{1} y q(y) d y=b q(b) b^{\prime}(a), \quad a<\alpha \\
a q(a)+\int_{a}^{1} q(y) d y=b q(b) b^{\prime}(a), \quad a>\alpha .
\end{gathered}
$$


Using (3.10) and (3.11) we see that the left side of (3.13) is $a q(a)-\int_{0}^{a} q(y) d y>0$ so that $b=b(a)$, defined by (3.12), has $b^{\prime}(a)>0$ (if $q(b)>0$ ) for $a<\alpha$. By (3.14), $b^{\prime}(a)>0$ for $a>\alpha$. Thus $b(a)=\phi^{-1}(a)$ increases from 0 to 1 so that $\phi(a)$ does also. Furthermore, we must have $b=b(a)<a$ for $0<a<1$ because if $b \geqq a$ in (3.12) then the right side is greater than the left since under (3.11) it is easy to see that

$$
2 \int_{0}^{a} x q(x) d x+a \int_{a}^{1} q(y) d y<\int_{0}^{a} x q(x) d x+\min \left\{\frac{a}{\alpha}, 1\right\} \int_{0}^{1} y q(y) d y .
$$

Thus under (3.9)-(3.11), (3.7) and (3.8) hold. Leaving aside the question of finding $q$ to satisfy (3.10) and (3.11) for some $0<\alpha<1$, suppose we then set $X_{3}^{\prime}=X_{3}=z$ where

$$
z=(1-\theta)(x \vee y)+\theta\left(x^{\prime} \vee y^{\prime}\right)
$$

and $\theta$ is independent of $(x, y)$ and uniform on $I=(0,1)$. Thus the measure space on which $X_{i}$ and $X_{i}^{\prime}$ are defined is $I^{3}=\{(x, y, \theta)\}$. Let $\mu$ be the measure induced by $\left(X_{1}, X_{2}, X_{3}\right)=(x, y, z)$ and $\nu$ be the measure induced by $\left(X_{1}^{\prime}, X_{2}^{\prime}, X_{3}^{\prime}\right)=\left(x^{\prime}, y^{\prime}, z\right)$. Clearly $\mu$ and $\nu$ satisfy (3.2) since $x \vee y<z<x^{\prime} \vee y^{\prime}$ and $\mu$ and $\nu$ have the same projections by (3.8) and the symmetry between $\left(x, x^{\prime}\right)$ and $\left(y, y^{\prime}\right)$.

We now verify that $\mu$ and $\nu$ have bounded densities. The density of $\mu$ for $x<y$ is from (3.5) and (3.6a) and the fact that $P\left(\theta\left(y^{\prime}-y\right) \in d z-y\right)=d z /\left(y^{\prime}-y\right)$,

$$
\begin{aligned}
\frac{\mu(d x, d y, d z)}{d x d y d z} & =q(y) \frac{P\left((1-\theta) y+\theta\left(x^{\prime} \vee y^{\prime}\right) \in d z\right)}{d z}=\frac{q(y)}{\left(x^{\prime} \vee y^{\prime}\right)-y} \\
& \leqq \frac{q(y)}{\phi(y)-y} .
\end{aligned}
$$

The density of $\nu$ for $x^{\prime}<y^{\prime}$ is as follows. If $\left(x^{\prime}, y^{\prime}\right)$ comes from $\left(x_{0}, y_{0}\right)$ with $x_{0}<y_{0}$, where $x_{0}<y_{0}$ denotes an event $A_{0}$ with indicator $\chi_{0}$, then from (3.5) and (3.6a),

$$
\begin{aligned}
\frac{\pi\left(A_{0} \cap\left(d x^{\prime}, d y^{\prime}, d z^{\prime}\right)\right)}{d x^{\prime} d y^{\prime} d z^{\prime}} & =\chi_{0} q(y) \frac{d x_{0} d y_{0}}{d x^{\prime} d y^{\prime}} \frac{P\left((1-\theta) y_{0}+\theta y^{\prime} \in d z^{\prime}\right)}{d z^{\prime}} \\
& =\chi_{0} q\left(y_{0}\right) \frac{y_{0}}{\psi\left(y_{0}\right)} \frac{1}{\phi^{\prime}\left(y_{0}\right)} \frac{1}{y^{\prime}-y_{0}} .
\end{aligned}
$$

However, $\left(x^{\prime}, y^{\prime}\right)$ may also come from $\left(x_{1}, y_{1}\right)$ with $x_{1}>y_{1}$, an event $A_{1}$ with indicator $\chi_{1}$. But

$$
\nu\left(d x^{\prime}, d y^{\prime}, d z^{\prime}\right) / d x^{\prime} d y^{\prime} d z^{\prime}=\pi\left(\left(A_{0} \cup A_{1}\right) \cap\left(d x^{\prime} d y^{\prime} d z^{\prime}\right)\right) / d x^{\prime} d y^{\prime} d z^{\prime}
$$

since $\left(x^{\prime}, y^{\prime}\right)$ comes from either a unique $\left(x_{0}, y_{0}\right)$ with $x_{0}<y_{0}$ or from a unique $\left(x_{1}, y_{1}\right)$ with $x_{1}>y_{1}$, or both. That is to say, if $\left(x^{\prime}, y^{\prime}\right)$ also comes from say $\left(x_{2}, y_{2}\right)$, then either $\left(x_{2}, y_{2}\right)=\left(x_{0}, y_{0}\right)$ or $\left(x_{2}, y_{2}\right)=\left(x_{1}, y_{1}\right)$ because $\phi$ is strictly monotonic. By (3.5) and (3.6b)

$$
\begin{aligned}
\frac{\pi\left(A_{1} \cap\left(d x^{\prime}, d y^{\prime}, d z^{\prime}\right)\right)}{d x^{\prime} d y^{\prime} d z^{\prime}} & =\chi_{1} q(x) \frac{d x_{1}}{d x^{\prime}} \frac{d y_{1}}{d y^{\prime}} \frac{P\left((1-\theta) x_{1}+\theta y^{\prime} \in d z^{\prime}\right)}{d z^{\prime}} \\
& =\chi_{1} q\left(x_{1}\right) \frac{1}{\phi^{\prime}\left(x_{1}\right)} \frac{x_{1}}{\psi\left(x_{1}\right)} \frac{1}{y_{1}^{\prime}-x_{1}} .
\end{aligned}
$$

By (3.18) and (3.17) and then (3.19) we have

$$
\frac{\nu\left(d x^{\prime}, d y^{\prime}, d z^{\prime}\right)}{d x^{\prime} d y^{\prime} d z^{\prime}} \leqq q\left(y_{0}\right) \frac{y_{0}}{\psi\left(y_{0}\right)} \frac{1}{\phi^{\prime}\left(y_{0}\right)} \frac{1}{\phi\left(y_{0}\right)-y_{0}}+q\left(x_{1}\right) \frac{x_{1}}{\psi\left(x_{1}\right)} \frac{1}{\phi^{\prime}\left(x_{1}\right)} \frac{1}{y_{1}^{\prime}-x_{1}} \text {. }
$$


But

$$
y_{1}^{\prime}-x_{1}=\frac{y_{1}}{x_{1}} \psi\left(x_{1}\right)-x_{1} \geqq \phi\left(x_{1}\right)-x_{1}
$$

since $y_{1}^{\prime}=y^{\prime}=\left(y_{1} / x_{1}\right) \psi\left(x_{1}\right)>x_{1}^{\prime}=x_{1}=\phi\left(x_{1}\right)$ and we are in case (3.6b). Thus $\nu$ will have a bounded density if and only if there is an $M$ for which

$$
q(y) \frac{y}{\psi(y)} \frac{1}{\phi^{\prime}(y)} \frac{1}{\phi(y)-y} \leqq M, \quad 0<y<1 .
$$

And, from (3.16), $\mu$ will have a bounded density if and only if there is an $M$ for which

$$
q(y) /(\phi(y)-y) \leqq M
$$

since both terms on the right in (3.20) have the same form after (3.21) is used in the denominator of the last term in (3.20).

It remains to show that there is $0<\alpha<1$ and $q(y), 0<y<1$, for which (3.10), (3.11), (3.22), and (3.23) hold for the $\phi$ determined by (3.8), as in (3.12)-(3.15). We choose in particular

$$
\alpha=2 / 5, \quad q(y)=y(1-y)^{2}
$$

and directly verify that (3.10) and (3.11) hold. It is clear from (3.13) and (3.14) that the only chance for $\phi(y)-y$ or $\phi^{\prime}(y)$ to be small is near $y=0$ or $y=1$. But near $a=0$, from (3.12), recalling $b=b(a)=\phi^{-1}(a),(3.11),(3.13)$, and (3.24),

$$
\int_{0}^{b} x^{2}(1-x)^{2} d x=2 \int_{0}^{a} x q(x) d x-a \int_{0}^{a} q(y) d y=\frac{a^{3}}{6}-\frac{a^{4}}{6}-\frac{a^{5}}{20}=\frac{b^{3}}{3}-\frac{b^{4}}{2}+\frac{b^{5}}{5} .
$$

Let $\approx$ stand for "bounded above and below by a constant multiple of." It follows that near $a=0, \phi^{\prime}(a) \approx 1, \phi(a)-a \approx q(a)$ and hence (3.22) and (3.23) hold near $a=0$. Near $a=1$, from (3.12), subtracting from (3.12) with $a=b=1$, and setting $1-a=\bar{a}$ and $1-b=\bar{b}$,

$$
\begin{aligned}
\int_{b}^{1} x^{2}(1-x)^{2} d x & =2 \int_{a}^{1} x q(x) d x-a \int_{a}^{1} q(y) d y \\
& =2 \int_{0}^{\bar{a}} x^{2}(1-x)^{2} d x-(1-\bar{a}) \int_{0}^{\bar{a}} x^{2}(1-x) d x \\
& =\frac{\bar{a}^{3}}{3}+\frac{\bar{a}^{4}}{12}-\frac{3 \bar{a}^{5}}{20}=\frac{\bar{b}^{3}}{3}-\frac{\bar{b}^{4}}{2}+\frac{\bar{b}^{5}}{5} .
\end{aligned}
$$

It follows that near $a=1, \phi^{\prime}(a) \approx 1, \phi(a)-a \approx(1-a)^{2} \approx q(a)$ and so (3.22) and (3.23) hold near $a=1$. This completes the proof that $\mu$ and $\nu$ have bounded densities.

The fact that there exist $\mu$ and $\nu$ with densities taking values only 0 and 1 follows easily from the following simple, powerful, and apparently new result, proved in [2]. This result says that if $f$ is any density in $\mathbb{R}^{n}$ with $0 \leqq f \leqq 1$, then there is a density $g$ with values in $\{0,1\}$ that has the same projections as $f$. This is proved in the case $n=2$ by observing that the necessary and sufficient condition for the existence of a bounded density with given marginals due to Strassen [11] is the same as the necessary and sufficient condition for the existence of a set with given marginals due to Lorentz [9] for $n=2$. The proof for general $n$ follows easily from the result for $n=2$. 
Final remarks. The first part of the proof of Theorem 3.2 shows that there is a fuzzy set with the same projections as $S_{0}$, where a fuzzy set is a function $f_{0}=f_{0}(x) \in[0,1]$. Indeed, if $\mu$ and $\nu$ are as in the proof with densities bounded by 1 (multiply by a small constant), we can set $f_{0}=\chi\left(S_{0}\right)-d \mu / d \lambda+d \nu / d \lambda$. Note that this is a much different statement than the trivial one that there is a bounded density $g_{0}(x)$ supported on the cube with the same projections as $\chi\left(S_{0}\right)$, i.e., as $S$; merely take

$$
g_{0}(x)=9 P_{1}\left(x_{1}\right) P_{2}\left(x_{2}\right) P_{3}\left(x_{3}\right) \text {, }
$$

where $P_{i}\left(x_{i}\right)$ is the projection of $S_{0}$ onto $x_{i}$,

$$
P_{i}\left(x_{i}\right)=\frac{1-x_{i}^{2}}{2} \text { for } i=1,2 ; \quad P_{3}\left(x_{3}\right)=x_{3}^{2} .
$$

The difference is that $g_{0}$ is not a fuzzy set because $g_{0}(x)>1$ at some points such as $g_{0}(0,0,1)=9 / 4$. It is interesting to note that there are analogous reconstruction problems in which a measure exists satisfying a given set of "moment" conditions, but no set exists. For example, it is impossible to find a set $A \subset[0,1]$ with Leb $(A \cap I)=$ $\frac{1}{2}$ Leb $(I)$ for every interval $I$, but there certainly is a measure (or fuzzy set), $\frac{1}{2} \lambda$, with this property. Related results are found in [10] (see also [3]), [5], and [11].

Appendix. Proof of Lemma 2.5. The bounded open sets $S_{1}$ and $S_{2}$ are constructed recursively: each $S_{i}$ is an infinite union of open unit cubes of size $2^{-k}$ for various values of $k$.

The basis of the construction is a configuration which fits inside a $3 \times 2 \times 2$ block made up of sets $T_{1}$ and $T_{2}$ each of which consists of three unit cubes. Let the block be $B=\{(x, y, z): 0 \leqq x \leqq 3,0 \leqq y \leqq 2,0 \leqq z \leqq 2\}$ and label each cube by its corner having the smallest coordinate values. The configuration is:

$$
\begin{aligned}
& T_{1}=\{(0,0,0),(1,0,0),(2,1,1)\} \\
& T_{2}=\{(0,1,0),(2,0,0),(1,0,1)\} .
\end{aligned}
$$

It is pictured in Fig. A.1, where the cubes in $T_{1}$ are labeled with a plus sign, and those in $T_{2}$ by a minus sign.

The basic configuration has three key properties:

(1) The sets $T_{1}$ and $T_{2}$ have the same projections on all three axes.

(2) $T_{1}$ contains a $2 \times 1 \times 1$ block in the lower left corner of the lower layer of $B$ and a $1 \times 1 \times 1$ block in the upper right corner of the upper layer of $B$.

(3) Any bad configuration for $T_{1}$ (or $T_{2}$ ) in $T_{1} \cup T_{2}$ must contain a point in every cube of $T_{1} \cup T_{2}$.

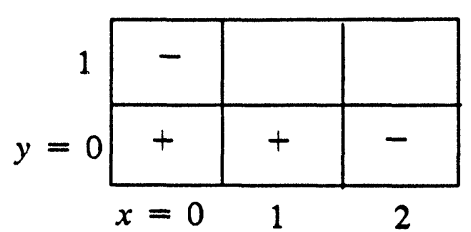

$$
z=0
$$

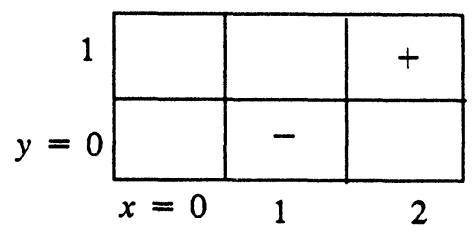

$z=1$

FIG. A.1. Basic configuration. 
The first two properties are clear. To prove the third property, we form an undirected graph whose vertices are the cubes in $T_{1} \cup T_{2}$ and where an edge represents a condition: "If a bad configuration includes a point in one vertex, then it necessarily includes a point in the other vertex." Such conditions are forced by the requirement that a bad configuration has equal projections for the subsets of $T_{1}$ and $T_{2}$. For example, if a bad configuration includes a point in the cube $(2,1,1)$ in $T_{1}$, then it must include a point in the cube $(1,0,1)$ in $T_{2}$, and vice versa, which is seen by examining its projection in the $z$-direction. For a point in $(2,1,1)$ in $T_{1}$ can only be matched in the $z$-direction by a point in $T_{2}$ having $1<z<2$, and all such points lie in the cube $(1,0,1)$. Proceeding in this way, we obtain the graph pictured in Fig. A.2, where edges are labeled by the direction of projection that verifies their occurrence. Since this graph is connected, it follows that a bad configuration includes every vertex.

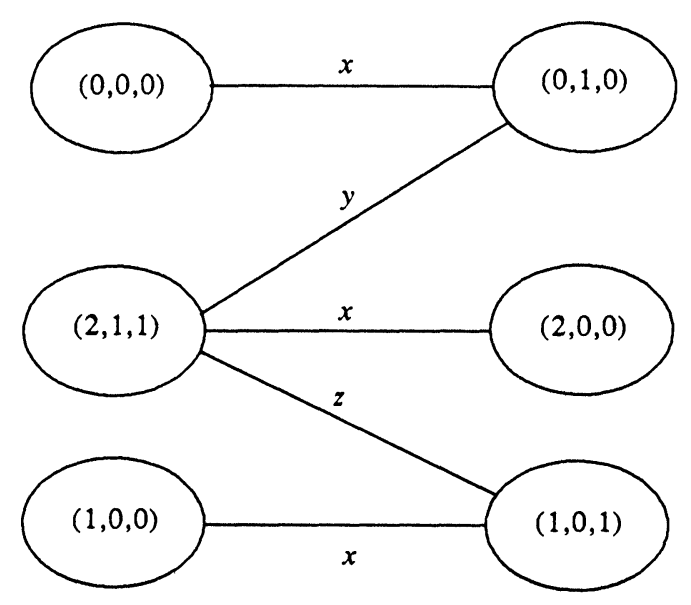

FIG. A.2. Graph associated to basic configuration.

We now begin the recursive construction. Construct a shrunken and rotated copy of the basic configuration by first scaling the first axis by $\frac{1}{2}$ and then permuting the axes cyclically to obtain a configuration in a $2 \times \frac{3}{2} \times 2$ block consisting of $1 \times \frac{1}{2} \times 1$ parallelepipeds. We place this $2 \times \frac{3}{2} \times 2$ block so that its lower left corner in its lowest level exactly overlaps the $1 \times 1 \times 1$ cube $(2,1,1)$ in $T_{1}$. The $1 \times 1 \times 1$ cube $(2,1,1)$ corresponds to the union of two cubes labeled $(0,0,0)$ and $(1,0,0)$ in the shrunken configuration. Let $\left(T_{1}^{\prime}, T_{2}^{\prime}\right)$ denote the analogues of $T_{1}$ and $T_{2}$ in the shrunken configuration. Our second set is:

$$
\begin{aligned}
& U_{1}^{\prime}=T_{1} \cup T_{2}^{\prime}-\{\text { cube }(2,1,1)\} \\
& U_{2}^{\prime}=T_{2} \cup T_{1}^{\prime}-\{\text { cube }(2,1,1)\} .
\end{aligned}
$$

Now we repeat this construction one more time, shrinking and rotating the basic configuration to obtain a $2 \times 1 \times \frac{3}{2}$ block containing the basic configuration as a set of $1 \times \frac{1}{2} \times \frac{1}{2}$ parallelepipeds, and adding it in to obtain:

$$
\begin{aligned}
& U_{1}^{\prime \prime}=T_{1} \cup T_{2}^{\prime} \cup T_{1}^{\prime \prime}-\{\text { two overlapping corner cubes }\}, \\
& U_{2}^{\prime \prime}=T_{2} \cup T_{1}^{\prime} \cup T_{2}^{\prime \prime}-\{\text { two overlapping corner cubes }\} .
\end{aligned}
$$

We call this configuration the composite configuration. 
Now we describe the composite configuration explicitly. For convenience we scale up the sets $U_{1}^{\prime \prime}, U_{2}^{\prime \prime}$ so that all cubes involved have side at least one, by expanding the $y$ and $z$ axes by a factor of 2 . When we do so, the resulting configuration $U_{1}, U_{2}$ is embedded in a $5 \times 6 \times 7$ block $B^{\prime}=\{(x, y, z): 0 \leqq x \leqq 5,0 \leqq y \leqq 6,0 \leqq z \leqq 7\}$. $U_{1}$ consists of a $2 \times 2 \times 2$ cube $A$, three $1 \times 1 \times 2$ parallelepipeds $B, C, D$, and a $1 \times 1 \times 1$ cube $E$ :
A. $(0,0,0),(0,0,1),(0,1,0),(0,1,1)$
$(1,0,0),(1,0,1),(1,1,0),(1,1,1)$
B. $(2,4,2),(2,4,3)$
C. $(2,3,4),(2,3,5)$
D. $(3,2,2),(3,2,3)$
E. $(4,5,6)$.

The set $U_{2}$ consists of three $1 \times 2 \times 2$ parallelepipeds $F, G, H$ and three $1 \times 1 \times 1$ cubes $I, J, K$, which are:
F. $(0,2,0),(0,3,0),(0,2,1),(0,3,1)$
G. $(2,0,0),(2,0,1),(2,1,0),(2,1,1)$
H. $(1,0,2),(1,0,3),(1,1,2),(1,1,3)$
I. $(3,5,4)$
J. $(4,4,5)$
K. $(3,4,6)$.

The composite configuration $\left(U_{1}, U_{2}\right)$ has three key properties:

(1) The sets $U_{1}$ and $U_{2}$ have the same projections on all three axes.

(2) $U_{1}$ contains a $2 \times 2 \times 2$ block in the lower left corner of its lowest two layers and a $1 \times 1 \times 1$ block in the upper right corner of its highest layer.

(3) Any bad configuration for $U_{1}$ (or $U_{2}$ ) in $U_{1} \cup U_{2}$ must contain a point in its highest cube $(4,5,6)$.

Here (1) and (2) are easily checked. Property (3) is proved directly using an argument similar to that given for the basic configuration. We use directed graphs whose vertices consist of several cubes, and whose directed edges indicate: "A bad configuration containing a point in some cube in the entering vertex must contain a point in one of the cubes in the vertex pointed to." The edges in the graph are constructed by arguments similar to those used for the basic configuration. Figure A.3 gives such graphs showing that any bad configuration must include a point in the cube $E$, thus proving property (3).

To describe the recursive construction of $S_{1}$ and $S_{2}$, we form an infinite set of copies of the composite configuration, shrunk by factors of $\frac{1}{2}$ at each step, so that the $k$ th composite configuration lies inside a $5 /\left(2^{k-1}\right) \times 6 /\left(2^{k-1}\right) \times 7 /\left(2^{k-1}\right)$ block $B_{k}$. These blocks are stacked successively so that the corner $E$ of the block $B_{k}$ exactly overlaps the corner $A$ of block $B_{k+1}$, as indicated in Fig. A.4.

Let $U_{1}^{(k)}, U_{2}^{(k)}$ denote the composite configuration in block $B_{k}$. The sets $S_{1}, S_{2}$ are given by:

$$
\begin{aligned}
& S_{1}=\left(\bigcup_{k=1}^{\infty} U_{1}^{(2 k-1)}\right) \cup\left(\bigcup_{k=1}^{\infty} U_{2}^{(2 k)}\right)-\{\text { all overlapped corners }\} \\
& S_{2}=\left(\bigcup_{k=1}^{\infty} U_{2}^{(2 k-1)}\right) \cup\left(\bigcup_{k=1}^{\infty} U_{1}^{(2 k)}\right)-\{\text { all overlapped corners }\} .
\end{aligned}
$$

If we let $\hat{U}_{1}$ denote $U_{1}-\{A\}-\{E\}$ then

$$
S_{1}=\{A\} \cup\left(\bigcup_{k=1}^{\infty} \hat{U}_{1}^{(2 k-1)}\right) \cup\left(\bigcup_{k=1}^{\infty} U_{2}^{(2 k)}\right) \quad S_{2}=\left(\bigcup_{k=1}^{\infty} U_{2}^{(2 k-1)}\right) \cup\left(\bigcup_{k=1}^{\infty} \hat{U}_{1}^{(2 k)}\right) .
$$



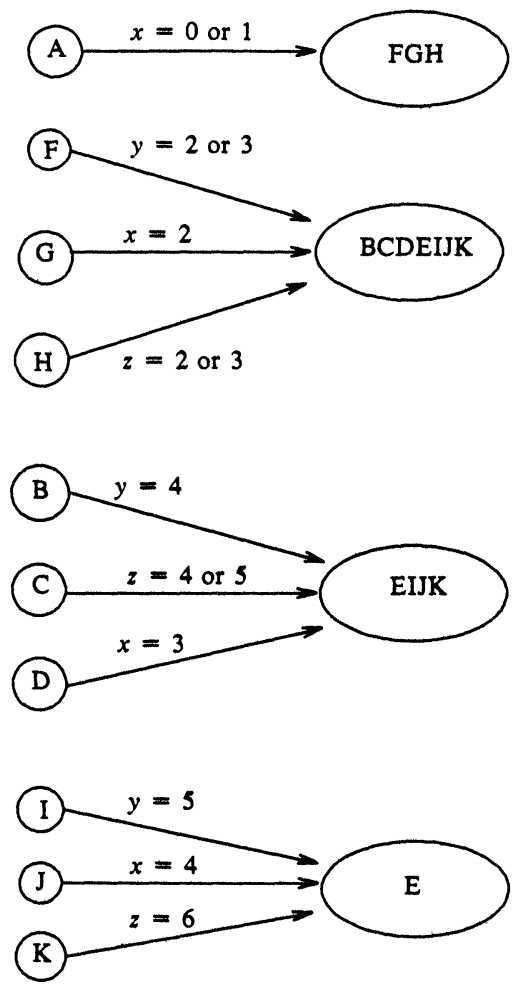

FIG. A.3. Directed graphs associated to composite configuration.

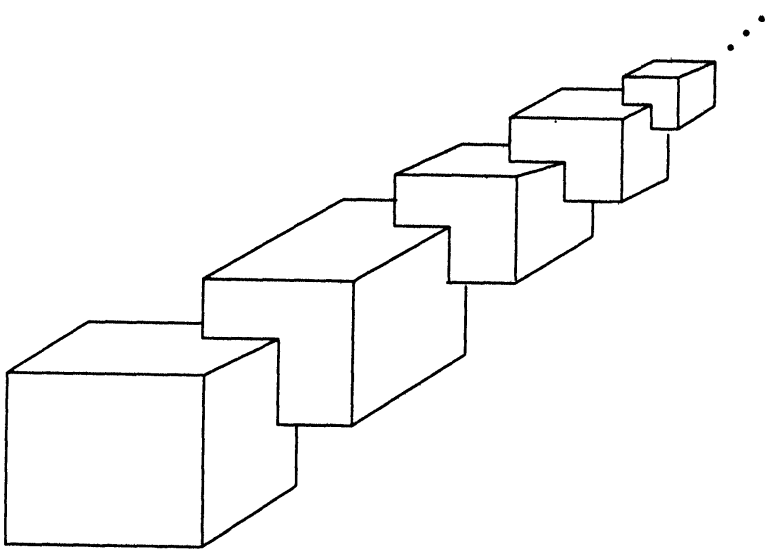

FIG. A.4. Recursive construction of blocks $B_{k}$.

It is easy to see that the sets $S_{1}$ and $S_{2}$ are bounded. Moreover, we show that:

(1) $S_{1}$ and $S_{2}$ have the same projections on all three axes.

(2) There exists no finite bad configuration for $S_{1}$ contained in $S_{1} \cup S_{2}$.

To show that (1) holds, we observe that

$$
\hat{S}_{1}=\left(\bigcup_{k=1}^{\infty} U_{1}^{(2 k-1)}\right) \cup\left(\bigcup_{k=1}^{\infty} U_{2}^{(2 k)}\right), \quad \hat{S}_{2}=\left(\bigcup_{k=1}^{\infty} U_{2}^{(2 k-1)}\right) \cup\left(\bigcup_{k=1}^{\infty} U_{1}^{(2 k)}\right)
$$


have the same projections on all three axes. Indeed, this holds by property (1) for the composite configuration and the fact that all sets in the union defining $\hat{S}_{1}$ (respectively, $\hat{S}_{2}$ ) are disjoint. The sets $\hat{S}_{1}$ and $\hat{S}_{2}$ are not disjoint, since their intersection $V$ is:

$$
V=\hat{S}_{1} \cap \hat{S}_{2}=\bigcup_{k=1}^{\infty} E^{(k)} \text {, }
$$

where $E^{(k)}$ denotes the corner cube $E$ in $U_{1}^{(k)}$, which is also the corner cube $A$ in $U_{1}^{(k+1)}$. Since $S_{1}=\hat{S}_{1}-V$ and $S_{2}=\hat{S}_{2}-V$, property (1) follows.

To prove property (2), let $\hat{U}^{(k)}=\hat{U}_{1}^{(k)} \cup U_{2}^{(k)}$, and call $\hat{U}^{(k)}$ the level $k$ points in $S_{1} \cup S_{2}$. We first prove the analogue of property (3) of the composite configuration, which is:

(3') Any bad configuration for $S_{1}$ in $S_{1} \cup S_{2}$ that contains a point in $\hat{U}^{(k)}$ must contain a point in $\hat{U}^{(k+1)}$.

This is proved by exactly the same graph-theoretic argument as in Fig. A.3, except that the vertex $E$ in that graph is interpreted to be "all points in $S_{1} \cup S_{2}$ in all levels $\geqq k+1$." The conclusion of property (3) that there must be a point in $E$ is replaced by the conclusion that there must be a point in the bad configuration at a higher level. This point must be on level $k+1$ because points on level $k$ and points on levels greater than or equal to $k+2$ do not have any projections that agree. So $\left(3^{\prime}\right)$ is proved. Finally, property ( $\left.3^{\prime}\right)$ implies property (2), because repeated applications of property $\left(3^{\prime}\right)$ produce at least one point in the bad configuration at all levels $\geqq k$. Hence the bad configuration contains infinitely many points.

Acknowledgments. We are grateful to S. H. Kahan for introducing us to the problem, to D. D. Sleator, H. S. Witsenhausen, and P. G. Doyle for technical observations, and to J. H. B. Kemperman, H. Kellerer, and A. Kuba for telling us about their own results.

\section{REFERENCES}

[1] P. C. Fishburn, J. C. Lagarias, J. A. Reeds, And L. A. Shepp, Sets uniquely determined by projections on axes II. Discrete case, preprint.

[2] S. Gutmann, J. H. B. Kemperman, J. A. ReEdS, AND L. A. ShePP, Existence of probability measures with given marginals, preprint.

[3] P. R. Halmos, The range of a vector measure, Bull. Amer. Math. Soc., 55 (1949), pp. 1015-1034.

[4] B. K. P. Horn, Robot Vision, MIT Press/McGraw-Hill, New York, 1985.

[5] H. G. Kellerer, Masstheoretische marginal probleme, Math. Ann., 153 (1964), pp. 168-198.

[6] J. H. B. KeMPERMAN, Sets of uniqueness and systems of inequalities having a unique solution, preprint.

[7] — On sets that are uniquely determined by a restricted set of integrals, preprint.

[8] A. KUBA AND A. VOLČIČ, Characteristics of measurable plane sets which are reconstructible from their two projections, Inverse Problems, 4 (1988), pp. 513-527.

[9] G. G. LORENTZ, A problem of plane measure, Amer. J. Math., 71 (1949), pp. 417-426.

[10] A. LyAPOUNOv, Sur les fonctions-vecteurs, complétement additives, Bull. Acad. Sci. USSR, 4 (1940), pp. $465-478$.

[11] V. STRASSEN, The existence of probability measures with given marginals, Ann. Math. Stat., 36 (1965), pp. 423-439.

[12] A. C. ZAANEN, Integration, North Holland, Amsterdam, 1967. 